\title{
Capítulo 2 Cadenas de suministro y AnyLogic
}

\section{Chapter 2 Supply chains and AnyLogic}

RODRÍGUEZ-PÉREZ, Ivonne†*

Universidad Autónoma del Estado de México. Centro Universitario UAEM Valle de México. Blvd. Universitario s/n Predio San Javier, Atizapán de Zaragoza, México, C.P. 54500

ID $1^{\text {er }}$ Autor: Ivonne Rodríguez-Pérez / ORC ID: 0000-0003-2982-2676, Thomson: B-2499-2016, CVU CONACYT ID: 57736

DOI: $10.35429 / \mathrm{H} .2020 .1 .15 .26$

\section{Rodríguez}

*irodriguezp@uaemex.mx

G. Gaviño \& R. Pérez. (AA.VV). Políticas, Casos de estudio, Técnicas de simulación y Programas de competencias en la educación de logística y cadena de suministro en México. Handbooks-CECORFAN-Mexico, Estado de México. 2020. 


\title{
Resumen
}

Actualmente las cadenas de suministros, otorgan ventajas competitivas a las empresas; por esta razón las Instituciones de Educación Superior, han puesto interés en el diseño y fortalecimiento de programas de estudio que desarrollen competencias en dicho ámbito. Derivado de esta necesidad se buscó, mediante el uso del método descriptivo las herramientas digitales (de simulación y optimización) que faciliten el aprendizaje de conceptos en logística y cadena de suministros, innovando así el proceso de enseñanza y aprendizaje de los alumnos. Considerando lo anterior, serán capaces en el ámbito laboral de mejorar las operaciones logísticas, contemplando la complejidad del negocio, los contextos cambiantes y las necesidades corporativas, usando herramientas de simulación y optimización. Estas han surgido en el sector logístico como una alternativa tecnológica para eficientar los procesos a partir de la previsión y el análisis de escenarios que contemplan las diferentes variables internas y externas de los negocios.

\section{Educación, Simulación, Cadena de suministros}

\begin{abstract}
Nowadays, supply chains give companies competitive advantages; For this reason, the Higher Education Institutions have put an interest in the design and strengthening of study programs that develop competences in this area. Derived from this need, the digital tools (simulation and optimization) that facilitate the learning of concepts in logistics and supply chain were sought through the use of the descriptive method, thus innovating the teaching and learning process of the students. Considering the above, they will be able in the workplace to improve logistics operations, contemplating the complexity of the business, changing contexts and corporate needs, using simulation and optimization tools. These have emerged in the logistics sector as a technological alternative to streamline processes based on forecasting and analyzing scenarios that contemplate the different internal and external variables of business.
\end{abstract}

\section{Education, Simulation, Supply chain}

\section{Introducción}

La complejidad en la administración de las cadenas de suministros ha crecido exponencialmente, imponiendo retos a los responsables de la toma de decisiones. Es necesario que los estudiantes que cursan materias en el ámbito de la Logística y Cadenas de Suministros desarrollen competencias necesarias para tomar decisiones efectivas. Para desarrollar dichas competencias los estudiantes deben entender de forma sistémica la naturaleza de los problemas implicados en la administración de las cadenas de suministro, y sobre todo los problemas estructurales que generan obstáculos de comunicación y coordinación en las redes logísticas.

Los profesores que imparten cursos en estas áreas también enfrentan el reto de proveer herramientas de aprendizaje que faciliten la comprensión de la complejidad por parte de los estudiantes, y que estos desarrollen las competencias necesarias para la toma de decisiones efectivas.

La intensificación del comercio mundial genera fuertes presiones sobre los sistemas de transporte y la infraestructura logística para manejar los elevados volúmenes de mercancías y otros bienes necesarios para la producción y el consumo.

La complejidad de los sistemas logísticos es tal que los enfoques tradicionales de aprendizaje son insuficientes para desarrollar el capital humano que se requiere. El enfoque sistémico que se puede lograr mediante la simulación, facilita la modelación de la complejidad y al mismo tiempo el desarrollo de los modelos, así como la utilización de los mismos, permitirá que los estudiantes asimilen la complejidad y desarrollen las capacidades necesarias para tomar decisiones efectivas en relación con el diseño y operación de las cadenas de suministros.

El enfoque de sistemas puede aportar elementos valiosos para la comprensión de la complejidad de las cadenas de suministros, permitiendo modelar características relevantes de los sistemas logísticos que escapan del alcance de los modelos de optimización tradicionales. 


\section{Cadena de suministro}

En algunas ocasiones parecen iguales, el concepto de logística con el de cadena de suministro. Ambos procesos están muy relacionados entre sí, de hecho hay quienes utilizan ambos términos indistintamente.

Council of Supply Chain Management Professionals (CSCMP) realiza una diferenciación y afirma que la logística implica el planeamiento y control de todas las actividades relacionadas con el suministro, fabricación y distribución de los bienes y servicios de una empresa; mientras que la cadena de suministro es la que eslabona a todas las compañías (proveedores de bienes y servicios y clientes), desde la adquisición de materias primas hasta la entrega del producto terminado. Se podría decir que han existido desde la antigüedad, cuando los orígenes de lo que se convertiría en cadenas de suministro lineales, movían los productos desde el origen hasta el punto de distribución.

En el camino, las redes comerciales y las rutas se multiplicaron, la comunicación se simplificó para ayudar a que los procesos de administración de la cadena de suministro fluyan de manera eficiente. Con el tiempo, estas redes de suministro evolucionaron para convertirse en modelos de gestión de la cadena de suministro (SCM) más sofisticados y complejos. Durante algún tiempo, estas soluciones de la cadena de suministro permanecieron aisladas, gestionadas por profesionales especializados que trabajaron para hacer que su proceso o sistema particular fuera eficiente. Con la aparición de las computadoras, y el uso de soluciones de SCM locales que automatizaron y optimizaron los procesos, permitieron que las cadenas de suministro aumentaran y así satisfacer las demandas de la globalización. La cadena de suministro es en un concepto con el cual las empresas mejoran las relaciones con los clientes y proveedores alcanzando una ventaja competitiva.

(Ballou, 2004), define cadena de suministro como un conjunto de actividades funcionales que se repiten a lo largo del canal de flujo del producto, mediante los cuales la materia prima se convierte en productos terminados y se añade valor al consumidor. Considerando lo anterior una cadena de suministro busca añadir valor al cliente, mejorar las relaciones con proveedores y aumentar las ganancias a los accionistas.

Según (Terrado, 2014), la cadena de suministro se compone de: logística de entrada, fabricación o logística interna, y distribución o logística de salida. Cuando estos tres elementos interactúan y coordinan actividades, se considera la creación y funcionamiento de la cadena de suministro.

\section{Características de la Cadena de suministro}

- $\quad$ Es dinámica e implica un flujo constante de información, productos y fondos en las diferentes etapas.

El cliente es parte primordial de las cadenas de suministro. El propósito 1 de las cadenas de suministro es satisfacer las necesidades del cliente.

- Una cadena de suministro típica puede abarcar varias etapas que incluyen: clientes, detallistas, mayoristas/distribuidores, fabricantes, proveedores de componentes y materias primas.

Cada etapa de la cadena de suministro se conecta a través del flujo de productos, información y fondos.

No es necesario que cada una de las etapas esté presente en la cadena de suministro.

- $\quad$ El diseño apropiado de la cadena de suministro depende de las necesidades del cliente como de las funciones que desempeñan las etapas que abarca.

La cadena de suministro se basa en tres etapas:

Figura 2.1 Fases de la cadena de suministro

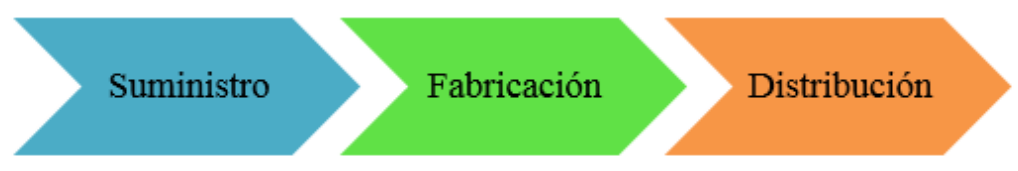


- Suministro: Se basa en el cómo, cuándo y dónde se obtienen los artículos que sirven como materia prima para la fabricación de cierto producto.

Fabricación: Como su nombre lo indica, se basa en convertir toda esa materia prima en productos terminados, los cuales serán útiles para determinada población.

Distribución: Este proceso se encarga de que dichos productos lleguen a las manos de los consumidores ¿Cómo? A través de una diversidad de redes de distribuidores, minoristas o comercios, Fig. 2.1.

Si bien las soluciones tradicionales de la cadena de suministro se enfrentaron a los primeros desafíos de una economía mundial con una mentalidad creciente, finalmente llegaron a sus límites. Los bienes y la información no podría cruzarse fácilmente de una parte de la cadena de suministro a la siguiente implicaba un esfuerzo manual y un alto potencial de errores. La visibilidad del inventario y la administración de transporte estaban limitadas por sistemas que no estaban integrados. Las organizaciones necesitaban más información con vistas en tiempo real en toda la cadena de suministro.

La llegada de internet cambió la forma en que funcionaban los sistemas tradicionales de la cadena de suministro. Un mercado más dinámico y centrado en el cliente exigía formas de trabajo más inteligentes y ágiles. Esperar que las mercancías fueran transportadas de un fabricante a una sala de exposición se había convertido en una opción para el consumidor.

\section{Gestión de la cadena de suministro (SCM)}

La Gestión de la Cadena de Suministro o en inglés Supply Chain Management (SCM), es definida por el Council of Logistics Management como la coordinación sistemática y estratégica de las funciones de negocio tradicional y las tácticas utilizadas a través de esas funciones de negocio, al interior de una empresa y entre los diferentes procesos de la cadena de suministro, con el fin de mejorar el desempeño en el largo plazo tanto de la empresa individualmente como de toda la cadena de suministro en general.

Se puede decir que la SCM tiene como objetivo garantizar las interacciones de los elementos logísticos, con el fin de que en la cadena de suministro se presente un flujo de productos e información óptimo, que permita la reducción de costos y el aumento de la satisfacción de los clientes. Cabe hacer mención, que el uso de las TIC ha facilitado que la SCM cumpla sus objetivos, debido que estas permiten el almacenamiento y transmisión de información logística, para la toma de decisiones. La SCM es un medio para que la empresa reduzca los costos y satisfaga las necesidades de los clientes, mejorando la competitividad.

SCM se puede dividir en 3 flujos que son:

Figura 2.1 Fases de la cadena de suministros

Flujo de cadena de suministro

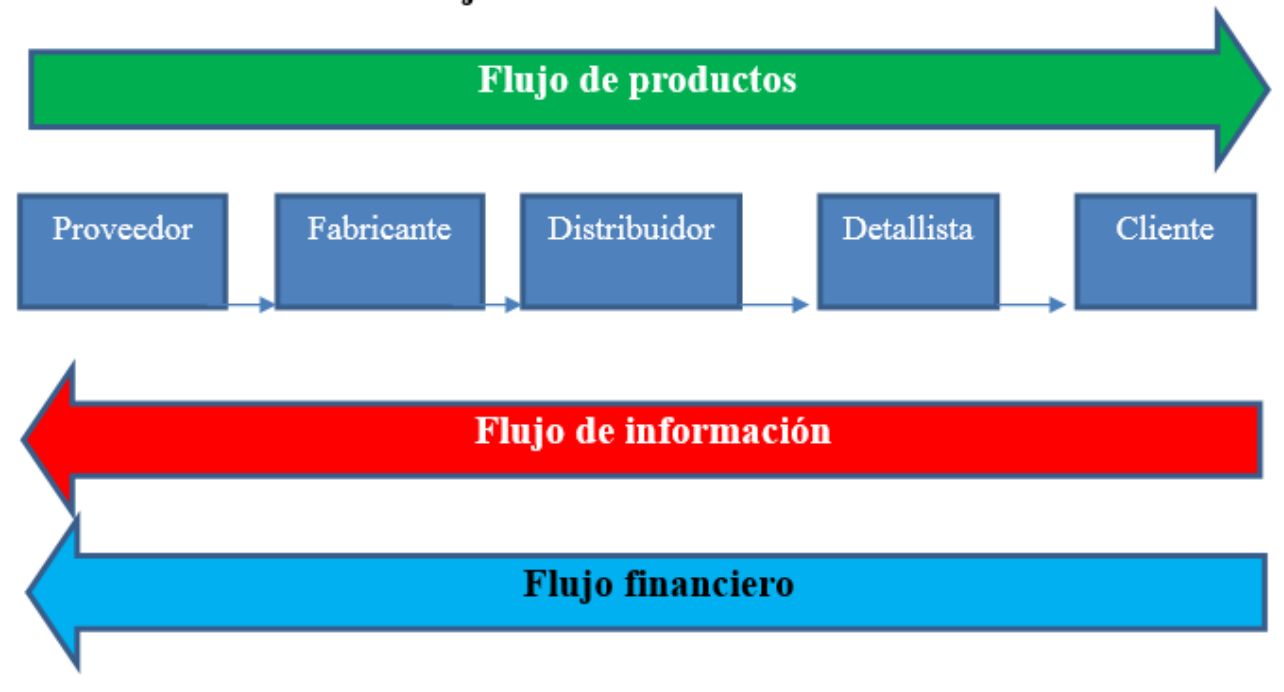

Fuente: Elaboración Propia 
- Flujo de productos: Este flujo engloba todo lo relacionado con la actividad que tiene la mercancía, abarcando el movimiento de productos que se tienen con los proveedores y los clientes, devoluciones que se puedan realizar y las necesidades de los servicios.

Flujo de información: Está vinculado con todos los procesos de comunicación de los pedidos y la información que se maneja con respecto a las entregas realizadas.

Flujo financiero: Abarca los calendarios de pago de la empresa. Fig. 2.2

\section{Importancia de una eficiente gestión de suministro}

Considerando lo anterior es conveniente tener en cuenta las razones del por qué es necesario contar con una eficiente gestión de la cadena de suministro:

- $\quad$ Fidelidad de los clientes: Como se tienen procesos productivos con eficiencia, se logran dar tiempos de respuesta casi inmediatos al cliente, mejorando los tiempos de entrega.

En consecuencia, los clientes siempre tendrán en mente a la compañía cuando tengan la necesidad de adquirir cierto producto, por ello, al lograr que la experiencia de compra sea satisfactoria, lo más probable es que repitan la compra.

- Se cuenta con un control más efectivo: Como todo el proceso se tiene bien definido, se logra conocer en tiempo real en que instante de la cadena de producción se encuentra el producto.

Esto garantiza que se tenga un mayor control con los proveedores, alcanzando una mayor cantidad de ofertas y un seguimiento a las fechas de entrada de los suministros, producción y demás procesos, los problemas diarios se reducen a su mínima expresión.

- $\quad$ Se reducen costos: Se logra que los costos se minimicen en un porcentaje considerable.

- Se potencia la empresa: Una eficiente gestión de la cadena de suministro no solo traerá a la empresa todos los beneficios descritos anteriormente, sino que además es capaz de hacer que la compañía sea líder en el sector en el cual se desenvuelve.

En otras palabras, el negocio despuntará en el mercado, haciendo que sea líder en el sector y manteniéndose por encima de tus competidores. Además, los clientes jugarán un papel importante, puesto que convertirán a la empresa en un referente.

- Todos trabajan como uno solo: Al tener claro cuáles son las funciones que desempeña cada integrante de la cadena, se comienza a trabajar de una forma organizada y sincronizada, haciendo que el producto llegue a los consumidores finales en las mejores condiciones posibles.

\section{TIC y SCM}

De acuerdo a la Asociación Americana de las Tecnologías de la Información, ITAA (como se citó en Correa y Gómez, 2008) las TIC son consideradas como "el estudio, el diseño, el desarrollo, el mantenimiento y la administración de la información por medio de sistemas informáticos, esto incluye no solamente la computadora, el medio más versátil y utilizado, sino también los teléfonos celulares, la televisión, la radio, etc".

Adicional a esta definición, es importante considerar la perspectiva otorgada por Cabrero (1998), en donde contempla que las TIC giran de forma interactiva en torno a la informática, las telecomunicaciones y la microelectrónica, permitiendo así nuevas realidades comunicativas. Las TIC, se han convertido en una ventaja competitiva para las empresas, ayudan en la transformación de los negocios, integración de procesos, mejora la productividad y la comunicación entre empresas, han influido positivamente en el funcionamiento de las cadenas de suministro, ya que se manejan en un ambiente globalizado, donde la información oportuna y de calidad se convierte en el mejor aliado. 
Los objetivos de las TIC en la SCM, son: a) Proporcionar información disponible y visible; b) Tener en un solo punto el acceso a los datos; c) Facilitar la toma de decisiones basadas en el hecho que se tiene información de toda la cadena de suministro y d) Permitir la colaboración entre los actores de la cadena de suministro. (Simchi-Levi ,2013)

Dentro de las TIC se encuentran diversas herramientas para procesos logísticos de cadenas de suministros, las cuales reducen los costos, mejora en el flujo de información y sincronización de procesos. Entre las que se encuentran las de simulación.

Dentro de las ventajas generales se abarca la optimización de la gestión administrativa interna y el mejoramiento al acceso de la información. El acceso a información confiable y de calidad facilita la ejecución de los procesos internos, dado que permite tomar decisiones en menores tiempos y con mayor exactitud; disminuyendo considerablemente la posibilidad de cometer errores (Ueki, Tsuji y Olmos, 2005).

Por otra parte, la aplicación de TIC ofrece la oportunidad de construir relaciones más cercanas con los clientes y facilita la obtención de nuevas oportunidades comerciales. Los clientes son la razón de ser de las empresas, es por esto que al conocer información a mayor profundidad sobre sus gustos, comportamientos y percepciones, se fortalece el vínculo comercial creado desde la primera venta, y se aumenta la posibilidad de crear nuevos lazos comerciales, gracias a la personalización de bienes y servicios (Apiyo, 2010).

Se considera otra ventaja el mejorar la competitividad en lo que se refiere a la gestión de materiales, control de calidad y fluidez de las operaciones. El desarrollo de aplicaciones y software ha permitido contar con una mayor trazabilidad de los productos a través de los múltiples procesos en los que se ven involucrados. Gracias a dicha trazabilidad, la ubicación de productos y el reconocimiento de errores a la hora de su producción o distribución, disminuye el desgaste de recursos humanos y técnicos en la corrección de fallas en los procesos (Evangelista et al., 2003).

Las TIC en la cadena de suministro proporcionan una reducción en los ciclos de tiempo, optimización de inventarios, disminución del efecto látigo, y un mejoramiento en la efectividad de los canales de distribución. Pero dichos beneficios pueden variar de acuerdo al método de implementación al interior de la organización. Por otra parte el uso de TIC se encuentra relacionado con la restructuración de procesos, y como tal la cadena de suministro puede ser vista como un proceso el cual requiere de cambios que pueden recibir soporte directamente de las TIC desarrolladas para la industria.

\section{Introducción a la simulación}

La simulación es la replicación de un sistema dado mediante el uso de métodos artificiales. Puede ser puramente analítico (mediante ecuaciones matemáticas) o computacional. Aunque es posible replicar las actividades de baja y mediana complejidad analítica, es extremadamente difícil construir modelos flexibles y obtener resultados precisos, por lo tanto, el uso de la computadora se convierte en esencial.

La simulación busca, entre otras cosas:

- $\quad$ Descubrir el comportamiento de un sistema.

- $\quad$ Postular teorías o hipótesis que expliquen el comportamiento observado.

- Utilizar esas teorías para predecir el comportamiento futuro del sistema, es decir los efectos que se producirían en el sistema, los cambios dentro de él o en su operación.

- Disminuir los costos asociados con la experimentación en el sistema real (prueba y error en el sistema real).

- $\quad$-Disminuir el riesgo de error. (Pinilla, 2004)

La simulación se puede utilizar para comprender mejor el impacto de decisiones específicas, mediante el uso de la computadora para sistemas reales. También es usada en centros educativos con el fin de desarrollar habilidades específicas, en las que los estudiantes controlan parte de las variables a través de interfaces de usuario. 
En las cadenas de suministro, esta herramienta es útil para entender la naturaleza y complejidad de los problemas interrelacionados y las variables que afectan. La integración de eslabones de una cadena tales como proveedores, fabricantes, mayoristas, distribuidores enfrenta algunos problemas que deben ser evaluados a fin de realizar acciones que permitan resolverlos adecuadamente

\section{Modelado}

El modelado es una forma con la que se pueden resolver problemas del mundo real. En muchos casos, no se puede experimentar con objetos reales para encontrar las soluciones adecuadas, ya que la construcción, destrucción, los cambios pueden ser demasiado caros, peligroso o simplemente imposibles. Este proceso supone la abstracción: incluir los detalles que se creen que son importantes y dejar de lado los que no lo son. El modelo es siempre menos complejo que el sistema original.

El uso de modelos como instrumentos de evaluación de alternativas tiene cada vez una mayor importancia ya que la mayoría de los procesos están sometidos al cambio continuo. Para poder tomar las decisiones idóneas es necesario saber cómo responderá el sistema ante una determinada acción. La simulación es el proceso de experimentar con un modelo.

Para ser útil, un modelo debe incorporar elementos de realismo y simplicidad. Por una parte, el modelo debe servir como una aproximación cercana al sistema real e incorporar la mayor parte de los aspectos del sistema. Por otro lado, el modelo no debe ser demasiado complejo para impedir su comprensión y manipulación. Cuando el modelo es relativamente simple, puede ser posible estudiarlo analíticamente mediante expresiones que describen el comportamiento de ciertos aspectos del sistema. Para sistemas más complejos, los enfoques analíticos suelen ser mucho más difíciles de realizar. En cambio, el sistema se analiza a menudo numéricamente mediante simulación por computadora, con el supuesto de que el sistema simulado es suficientemente parecido al sistema real para sacar conclusiones válidas sobre el último. Fig. 2.3

Figura 2.3 Modelado de la simulación y análisis

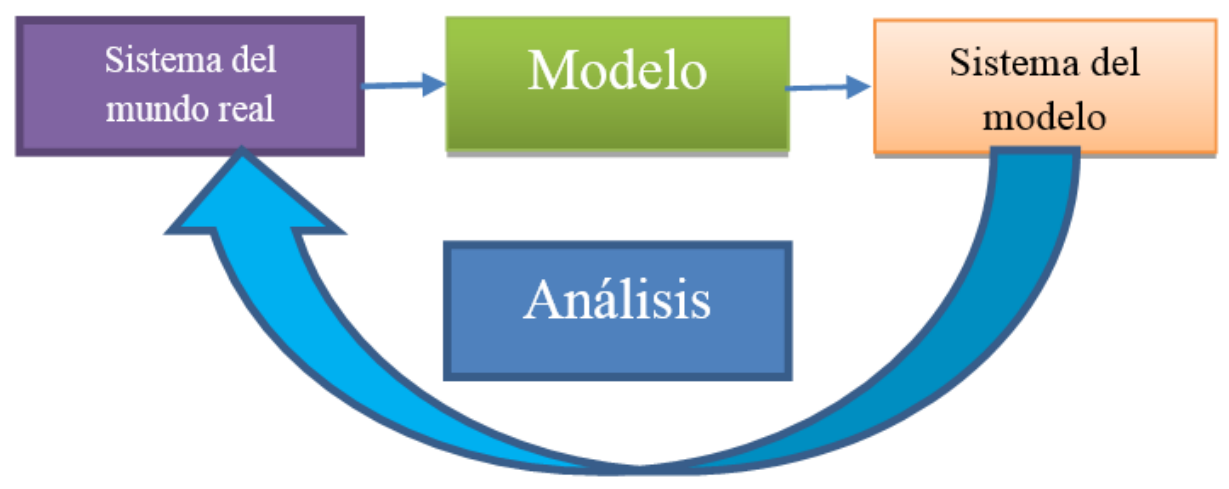

Fuente: Kroese, et al, 2011

Los modelos de simulación pretenden imitar el comportamiento de los sistemas de la vida real. Un sistema está formado por una colección de entidades u objetos que interactúan formando un todo complejo (Kroese, et.al, 2011). Los modelos se pueden clasificar en función de la manera en que las variables evolucionan en el tiempo.

Tiempo Continuo: Las variables evolucionan continuamente en el tiempo. Generalmente se representan mediante ecuaciones diferenciales.

Tiempo Discreto: Las variables sólo pueden cambiar en determinados instantes de tiempo.

Eventos Discretos: Las variables pueden cambiar en cualquier momento, pero sólo puede haber números finitos de cambios en intervalos de tiempo finitos. 
La reutilización de modelos y de componentes parece tener cierto potencial para ayudar a acelerar y facilitar el desarrollo de las simulaciones, especialmente si los componentes podrían ser compartidos entre desarrolladores de modelos.

Otra vía para cambiar el desarrollo de los modelos de simulación es automatizar el proceso. El modelador podría proporcionar una descripción y un intérprete convertiría esto en código de modelo ejecutable.

El uso del modelo también implica tomar una serie de cuestiones como establecer las condiciones iniciales, decidir cuánto tiempo se deja correr la simulación, número de repeticiones, entre otros. Existen métodos que podrían utilizarse para guiar a un usuario en todas estas áreas, pero hay pocas evidencias de que estos métodos estén implementados en softwares comerciales de simulación. Esto podría ser abordado mediante el desarrollo de software para guiar la simulación de usuarios o incluso automatizar aspectos del proceso.

Los modelos de simulación requieren de herramientas especiales de software que utilizan lenguajes específicos para la simulación. Es muy complejo realizar un modelo de simulación, pero su tiempo y esfuerzo serán recompensados cuando ofrezca un análisis de alta calidad de un sistema dinámico.

Actualmente se pueden encontrar una gran variedad de programas de simulación para la mayoría de industrias, manejando proyectos complejos, y a gran escala, relacionados con la fabricación, procesos, logística, distribución, almacenamiento o sistemas de servicios.

Actualmente existen en el mercado una gran variedad de software de modelado, simulación y virtualización de procesos. La llegada de la tecnología 3D ha conseguido que estos sean más sencillos e intuitivos.

Entre los más conocidos se encuentran:

Promodel: es una tecnología de simulación de eventos discretos que se utiliza para planificar, diseñar y mejorar nuevas o existentes de fabricación, logística y otros sistemas operativos. Representa con precisión los procesos del mundo real, incluyendo su variabilidad inherente y las interdependencias, con el fin de realizar un análisis predictivo de los cambios potenciales.

Arena: ha sido durante 30 años el software de simulación de eventos discretos líder en el mundo. La simulación de eventos discretos describe un proceso con un conjunto de eventos únicos, específicos en el tiempo. Estos modelos flexibles, basados en la actividad pueden ser utilizados eficazmente para simular casi cualquier proceso.

Anylogic es considerada como una herramienta de simulación de última generación. Un software que ofrece un sistema flexible, orientado a objetos para el desarrollo de simulación de eventos discretos, modelos basados en agentes y de dinámica de sistemas. Los modelos de Anylogic se pueden exportar como applets Java o aplicaciones Java autónomas, sin necesidad de un entorno de desarrollo Anylogic en funcionamiento.

\section{Anylogic}

Este software pertenece a Anylogic Company, empresa que creó el software en 2000 en Rusia. Con su desarrollo se ha conseguido que con una única herramienta se pueda englobar la heterogeneidad de los sistemas de negocios. Trabaja en un entorno Java, que tiene la ventaja de tener una extensibilidad ilimitada, además de poder importar códigos propios y bibliotecas y fuentes de datos externas. Se puede desarrollar el proyecto en cualquier lugar tan solo generando una aplicación Java.

Cuenta con un amplio conjunto de funciones de distribución estadística que proporciona una buena plataforma para la simulación de la incertidumbre. También cuenta con una función de apoyo a simulaciones de Montecarlo. 
Gracias a la simulación 3D, se puede diseñar cada objeto dentro de una misma cadena con diferentes detalles. Se basa en el modelado de agentes, y su uso abarca un campo muy variado, pudiendo servir para aplicaciones en cadenas de suministros y logística, mercados y competencia, ferrocarriles, salud, flujos de personas, militares, planificaciones y gestiones estratégicas o procesos sociales (The Anylogic Company, 2017).

AnyLogic es una herramienta de simulación que soporta las metodologías más comunes: Dinámica de Sistemas (DS), Sistemas de eventos discretos (SED), y el modelado basado en agentes (multy agent systems, MAS).

Utiliza con un lenguaje de simulación flexible, modelando diferentes sistemas complejos y heterogéneos como sistemas empresariales, económicos e incluso sociales, entre otros. AnyLogic permite construir modelos de forma modular, jerárquica e incremental generando modelos complejos y grandes. Además, ofrece un conjunto de objetos que permiten incorporar elementos de simulación preconstruidos de forma rápida.

Trabaja en Java y puede utilizarse de forma predeterminada o personalizada incluyendo bibliotecas o fuentes de datos externas. Las applet de Java pueden ejecutar el modelo en cualquier lugar sin necesidad de tener instalado el software el simulador.

Las funciones de distribución estadísticas predefinidas, permiten simular sistemas definidos por incertidumbre, también cuenta con paquetes de optimización para aplicarlos a diversos enfoques de simulación. Dispone de funciones de animación para generar modelos con un entorno de simulación interactivo y muy visual.

La interfaz gráfica, las herramientas y los objetos de biblioteca de AnyLogic permiten modelar diversas áreas como: cadenas de suministro y logística, marketing y competitividad, producción, almacenamiento, proyectos, procesos de negocio, transporte y gestión de activos, sistemas de servicios, entre otros. Figura 2.4 .

Figura 2.4 Ejemplo de interfaz gráfica

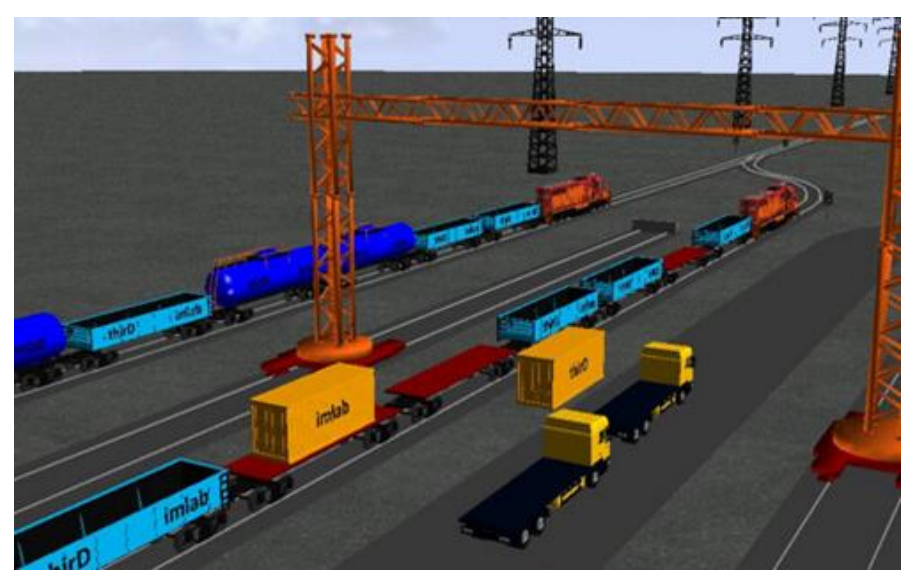

Fuente: Plantilla de Anylogic

La modelación y simulación de sistemas logísticos puede requerir la utilización de diferentes paradigmas de simulación además del enfoque de Simulación Dinámica de Sistemas (Systems Dynamics). Cuando se trata de simular el comportamiento de la cadena de suministros interna de una planta puede ser adecuado utilizar el paradigma de Simulación de Eventos Discretos. Si se trata de simular el comportamiento de los flujos de productos y de fondos a lo largo de los diversos eslabones de las redes de suministro puede ser más adecuado el paradigma de Simulación Dinámica.

La herramienta de simulación que permite la utilización e incluso la integración de diferentes paradigmas de simulación en un solo ambiente es AnyLogic. La herramienta que mejor cumple con este requerimiento. (Borshchev, 2014) 
Además de la gran ventaja que provee AnyLogic al permitir la integración de múltiples paradigmas de simulación, tanto la construcción de los modelos como la utilización interactiva de los mismos se facilita por su amigable interfaz gráfica. El diseño de los modelos es intuitivo y visual.

Los componentes más simples de un modelo se utilizan fácilmente tal y como vienen preconstruidos en el programa, la modelación de estructuras más complejas no es tan simple, pero la herramienta provee la capacidad de modelarlas mediante el desarrollo de código en Java dentro de la misma interfaz de AnyLogic.

El comportamiento dinámico de las cadenas de suministro es modelado con la ayuda de elementos de animación visuales, que permiten observar tanto el movimiento físico de materiales, el comportamiento de variables relevantes a través del tiempo, como pueden ser los valores de demanda, inventarios, transferencias de materiales, entre otros.

Para observar el efecto de las decisiones en la cadena de suministros, Anylogic provee elementos para el control interactivo de parámetros del sistema, de manera que a través de botones y deslizadores, el usuario puede alterar los valores de los parámetros y observar así el efecto de sus decisiones sobre otras variables del sistema. Ejemplo de la pantalla de Anylogic.

AnyLogic es importante para la toma de decisiones y simulación, permitiendo comparar escenarios colaborativos frente a los no-colaborativos. Figura 2.5.

Figura 2.5 Ejemplo de interfaz gráfica

\section{Ventajas}

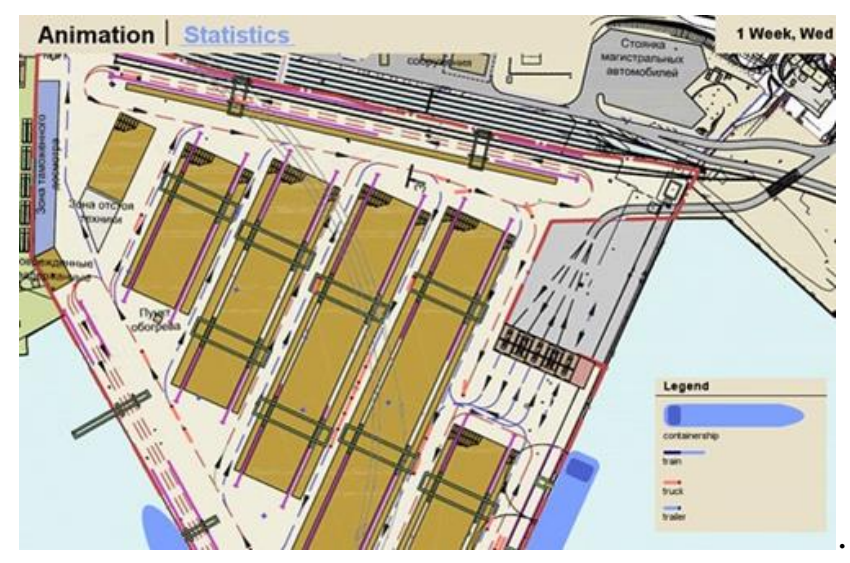

Fuente: Plantilla de Anylogic

Realizar una simulación permite:

- Analizar sistemas y encontrar soluciones donde los métodos tales como cálculos analíticos y de programación lineal falla.

- $\quad$ Es más fácil de desarrollar un modelo de simulación que un modelo analítico.

- $\quad$ En un modelo de simulación, se puede medir los valores y se pueden añadir mediciones y análisis estadísticos en cualquier momento.

- $\quad$ Los modelos de simulación son mucho más convincentes que las hojas de cálculo.

\section{Conclusiones}

El modelado y la simulación puede proporcionar un medio muy eficaz para evaluar el diseño de un nuevo proceso de fabricación o modificaciones propuestas a procesos existentes. El principal estándar que hay que establecer es la metodología. Actualmente, se realizan estudios para analizar y mejorar la eficiencia y eficacia de organizaciones, sistemas y procesos de fabricación. La solución a este problema será construir una serie de plantillas con las que todas las empresas puedan trabajar. Crear bloques de construcción únicos en cada empresa e incorporarlos a las plantillas prediseñadas, con el fin de ahorrar costos económicos y pérdidas de tiempo innecesarias. 
Las interfaces de datos estándar deben ser neutrales e independientes del proveedor para almacenarlos y que otros usuarios puedan adquirirlos, lo que mejoraría la accesibilidad a la simulación. Estos datos también serven para el desarrollo de más modelos reutilizables y datos de referencia entre empresas, proveedores, fabricantes y consultores. Por último, también beneficiarían a mejorar la sostenibilidad al crear datos de referencia de los procesos, como consumo de energía, satisfacción laboral, etc. (McLean, 2009).

El uso de simuladores como herramientas de aprendizaje no es algo nuevo en el ámbito educativo (Damassa,2014), sin embargo, la aplicación de los simuladores en el ámbito específico de Logística y Cadenas de Suministros cobra especial relevancia en esta segunda década del siglo XXI. La complejidad de las cadenas de suministros ha crecido de manera extraordinaria debido a la globalización de la producción y del consumo.

Aunado a lo anterior, hay otros temas que complican aún más la administración de las cadenas de suministros como son la incidencia de desastres naturales que incrementan los riesgos logísticos, la preocupación por la conservación del medio ambiente, la sofisticación de los consumidores y de los mercados entre otros factores.

Esta creciente complejidad exige que el capital humano involucrado en la administración de los sistemas logísticos actuales desarrolle habilidades diferentes a las que se requerían hace apenas unos años. Se requiere la comprensión integral de sistemas complejos y la capacidad de tomar decisiones bajo un ambiente de incertidumbre.

Los simuladores contribuyen a desarrollar las competencias necesarias para la toma de decisiones efectivas en el ámbito de las cadenas de suministros. Los modelos desarrollados reproducen parte de la complejidad de los sistemas logísticos actuales y permiten la toma de decisiones que afectarán el desempeño del sistema simulado. De esta manera el estudiante puede tomar decisiones en un ambiente seguro, observar las consecuencias de sus decisiones, y entender el comportamiento del sistema que se está modelando. Los estudiantes aprenden a tomar decisiones con información limitada, a establecer y probar hipótesis lógicas, y a reaccionar de manera ágil ante los cambios que ocurren en el ambiente.

La complejidad de las organizaciones en cualquier ámbito del quehacer humano se incrementa, complicando por lo tanto la toma de decisiones.

El perfil de los egresados deber incluir la capacidad de entender el comportamiento de sistemas complejos y tomar decisiones bajo un ambiente de incertidumbre. Para desarrollar dichas competencias, los simuladores de sistemas complejos tienen gran relevancia.

\section{Referencias}

Apiyo, R. (2010). Role of ICT Tools in Supply Chain Performance. 3-17.

Ballou, Ronald H. (2004). Logística, Administración de la cadena de suministro. México, Pearson Educación.

Chopra, Sunil., \& Petre Meindl, P. (2008). Administración de la cadena de suministro estrategia, planeación y operación. México: Pearson Educación

Cabrero, Julio. (1998). Impacto de las nuevas tecnologías de la información y la comunicación en las organizaciones educativas. Grupo Editorial Universitario. (pp. 197-206)

Campuzano, Josefa (2011). Supply Chain Simulation. A System Dynamics Approach for Improving Performance (p. 106). Springer London Dordrecht Heidelberg New York.

Council of Supply Chain Management Professionals (2014). Documento. Disponible en http://cscmp.org/about-us/supply-chain-management-definitions

Correa, Alejandro., Gómez, Ricardo. (2009). Tecnologías de la información y comunicación en la gestión de almacenes. Revista Avances en Sistemas e Informática, 6, 113-118 
Damassa, David A.; Sitko, Toby D. Simulation Technologies in Higher Education: Uses,Trends, and Implications. ECAR Research Bulletin 3, 2010. Boulder, Co. USA.

Evangelista, et.al. (2010). ICT Innovation Diffusion in Small Logistics Service Providers:an Empirical Study. International Journal of Logistics and Transport, 53-70

Gunasekarana, Laib. K. Andt (2008). Responsive supply chain: A competitive strategy in a networked economy. Omega, Vol, 36

Kroese, D. P., Taimre, T., \& Botev, Z. (20 de Septiembre de 2019). Handbook of Monte CarloMethods. Disponible en: http://onlinelibrary.wiley.com/doi/10.1002/9781118014967.ch7/pdf

McLean, C. (2009). Manufacturing Simulation: The Need for Standard Methodologies, Models, and Data Interfaces. National Workshop on Challenges to Innovation Advanced Manufacturing, (págs.8-9).

Pilot, Flyin J. (2016). Supply Chain. Disponible en:http://www.programaempresa.com/em

Poler, Raúl, Mula, Josefa, Díaz-Madroñero, Manuel. (2013). ADGIP: Una herramienta docente para el modelado y resolución de árboles de decisión. Modelling in Science Education and Learning.presa/empresa.nsf/paginas/D0407C086A64D097C125705B00322388?OpenDocument

Pricewaterhousecoopers. Manual Práctico de Logística. Available:http://www.programaempresa.com/empresa/empresa.nsf/paginas/BA43A3DF9ED296C1C12 5705B0024E380?OpenDocument.

Spagnoletti, Paolo and Edoardo, D'Atri and Alessandro , D'Atri (2013) Managing Decision Making in Supply Chains and Value Networks: the Beer Game Evolution. International Journal of ElectronicCommerce Studies.

Sparling, David. (2012).Simulations and supply chains: Strategies for teaching supply chain management. Supply Chain Management 7(5):334-342.

Terrado, Alejandro (2014). La Cadena de Suministro, Argentina.

The Anylogic Company. (2019). Anylogic, Multimethod Simulation Software. Disponible en http://www.anylogic.com

Ueki, Y., Tsuji, M., \& Olmos, R. (2005). Tecnología de la información y las comunicaciones (TIC) para el fomento de las pymes exportadoras en América Latina y Asia oriental. Naciones Unidas, 10-12

Urzelai, Inza, (2008). Manual Básico de Logística Integral, Díaz de Santos, España. 